\title{
Assessments of the quality systems of pharmaceutical distributors: a remote approach to be applied in times of COVID-19 and beyond
}

\author{
Anthony Bourasseau ${ }^{1}$, Laurine Lavergne ${ }^{2}$ and Raffaella Ravinetto ${ }^{3^{*}}$ (D)
}

\begin{abstract}
Background: Adequate quality systems throughout pharmaceutical supply chains are crucial to protect individuals and communities from substandard and falsified medical products. Thus, pharmaceutical distributors are regularly assessed by qualified experts. Since the COVID-19 pandemic has forced a suspension of normal activities, remote assessments via videoconferencing may represent a temporary alternative to on-site audits. We exploratorily evaluated the feasibility of remote assessments of pharmaceutical distributors, located in a low- or middle-income country hard-to reach during the COVID-19 pandemic.
\end{abstract}

Methods: We conducted pilot remote assessments of four conveniently selected distributors. The expert was remotely connected via videoconference, and supported by an in-country assessment facilitator (ICAF), who had received ad hoc training and was present at the assessed facility. First, the remote expert assessed the quality assurance (QA) activities and rated their compliance with the standards of the World Health Organization Good Storage \& Distribution Practices (GSDP), as per routine practice. Second, s/he assessed the completeness, clarity and accuracy of data collected remotely, first per distributor, and then in aggregated form.

Results: Data completeness was assessed by the expert as excellent, while clarity and accuracy were good. Overall data quality (a combination of completeness, clarity and accuracy) was good, with no major differences across QA activities, nor across distributors. Contextual limitations included poor internet connection, language barriers, and distributors' lack of familiarity with QA terminology.

Conclusions: Our findings are exploratory and cannot be extrapolated to other contexts, nor to other types of audits. Nonetheless, this pilot experience suggests that a well-planned remote assessment of pharmaceutical distributors, conducted with the support of a well-trained onsite ICAF, can provide data of acceptable quality, allowing to assess GSDP-compliance and to make temporary decisions about licensing or purchasing. Purchasers and policy makers should read the results of remote assessments in light of their intrinsic limitations. At the moment, onsite assessments remain the gold standards, but this could change in the longer term, with improved information technology and in light of the need to avoid unnecessary travels. Furthermore, remote assessments could be considered for routine prescreening candidates for on-site assessments, and for targeted follow-up of on-site assessment.

\footnotetext{
*Correspondence: rravinetto@itg.be

${ }^{3}$ Department of Public Health, Institute of Tropical Medicine,

Nationalestraat 155, 2000 Antwerp, Belgium

Full list of author information is available at the end of the article
}

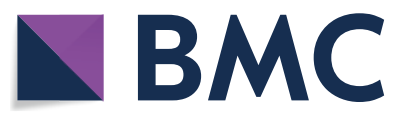

(c) The Author(s) 2021. Open Access This article is licensed under a Creative Commons Attribution 4.0 International License, which permits use, sharing, adaptation, distribution and reproduction in any medium or format, as long as you give appropriate credit to the original author(s) and the source, provide a link to the Creative Commons licence, and indicate if changes were made. The images or other third party material in this article are included in the article's Creative Commons licence, unless indicated otherwise in a credit line to the material. If material is not included in the article's Creative Commons licence and your intended use is not permitted by statutory regulation or exceeds the permitted use, you will need to obtain permission directly from the copyright holder. To view a copy of this licence, visit http://creativecommons.org/licenses/by/4.0/. The Creative Commons Public Domain Dedication waiver (http://creativeco mmons.org/publicdomain/zero/1.0/) applies to the data made available in this article, unless otherwise stated in a credit line to the data. 
Keywords: Quality of medicines, Pharmaceutical distributor, Supply chain, Remote audit, Distant assessment, Videoconferencing, Information technology, Travel restrictions, Developing countries, COVID-19

\section{Background}

The Sustainable Development Goal 3.8 aims at universal health coverage (UHC), including quality and affordable essential medicines and vaccines for all. There is increasing evidence that adequate regulatory oversight and medicines quality assurance mechanisms throughout the supply chain are crucial to protect individuals and communities from substandard and falsified medical products, and that they are strictly interconnected with the goals of UHC [1]. Unfortunately, the quality systems of pharmaceutical distributors in low-income and middleincome countries (LMICs) tend to be weak [2], and insufficient to prevent and detect the supply of poor-quality medicines [3]. Furthermore, the weaknesses of National Regulatory Authorities (NRA) hinder the implementation of stringent regulatory supervisions along the supply chain in many LMICs. To correct this situation, the World Health Organization (WHO) started a Global Benchmarking of Regulatory Systems for evaluating the national regulatory systems through a comprehensive and systematic benchmarking $[4,5]$. While achieving stringency of all NRAs remain the ultimate goal of stakeholders in pharmaceutical systems, short- and middle-term strategies are needed to secure the quality of medicines and other health products procured in poorly regulated contexts [6].

Twenty-six non-for-profit purchasers of medicines and other health products are currently members of QUAMED. QUAMED stands for "Quality Medicine for All". It is a humanitarian alliance that aims to improve access to quality medicines, by raising awareness among key players in pharmaceutical supply systems, and by reinforcing the quality assurance and supply policies of its members [7]. Among its activities, QUAMED conducts assessments of pharmaceutical suppliers, and it advices its own members for the selection of reliable local suppliers. The assessments are conducted according to the standards of the WHO Good Manufacturing Practices (GMP) [8], for manufacturers of finished pharmaceutical products, of the WHO Model Quality Assurance System (MQAS) [9], for procurement agencies/distributors that prequalify their own sources and suppliers, and of the WHO Good Storage \& Distribution Practices (GSDP) [10], for "technical visits" of distributors that do not prequalify their own sources and suppliers.

Pharmaceutical assessments traditionally require the on-site presence of a qualified expert, who carries out first-hand observations of policies, procedures, records and practices at the assessed facilities. Unfortunately, the COVID-19 pandemic has forced a partial suspension of normal activities, due to the restrictions on international travels. Under these circumstances, remote assessments could represent a temporary alternative to the on-site assessments. To the best of our knowledge, there are no explicit restrictions in the $\mathrm{WHO}$ and regulatory guidance to conduct remote audits or inspections, but there is no detailed guidance either. For the European Medicines Agency (EMA), a distant assessment is an "assessment of the compliance of a site (...) on the basis of documents and interviews and supported by technology for communicating, accessing systems, sharing and reviewing documents and other information, without the inspectors being physically present at the sites, where the activities subject to the assessment have taken place and where the inspection would ordinarily be hosted" [11]. According to EMA, "on-site inspections should be conducted when circumstances permit following the distant assessment", and inspectors should make "a case-by-case decision on whether a distant assessment is considered appropriate and feasible. The criticality of the product should be taken into consideration". Furthermore, the optimal communication platform could include a live videoconference platform with break-out rooms/conferences and screen sharing, smart glasses or other mobile cameras which can be interfaced to the videoconference platform, and access to a secure cloud server to share documents."[11]. EMA also published a "Guidance on remote Good Clinical Practices inspections during the COVID-19 pandemic" [12], and the EU Medical Device Coordination Group published a detailed "Guidance on temporary extraordinary measures related to medical device Notified Body audits during COVID-19 quarantine orders and travel restrictions" [13]. For the United States Food and Drug Administration (US-FDA), a remote audit is an "audit performed off-site through the use of information and communication technology" (synonyms are eAudit, and virtual audit)[14]. At the beginning of the COVID19 pandemic, the US-FDA postponed on-site GMP and GSDP inspections, before adjusting their strategy based on the risks [15]. The US-FDA's Medical Device and Single Audit Program started a Remote Auditing pilot program, to gauge the viability of remote Device Marketing Authorization and Facility Registration Process [16], but to the best of our knowledge, no guidance has been issued for remote pharmaceutical inspections. Conversely, a few stakeholders in the private sector developed 
some guidance. For instance, Mark Durivage, from Quality Systems Compliance LLC, illustrated the pros and cons of remote GMP audits versus on-site audits [17], while Freyr Solutions linked remote "desktop" audits to the levels of risk in a self-speaking infographic [18].

Overall, even if video conferencing is today accepted practice in several sectors, there is little public information on the use of Information Technology (IT) and video-conferencing tools for the remote assessment of pharmaceutical facilities. In addition, scientific literature provides little or no guidance on whether this would be feasible. There is an urgent need to address this question, and to understand which factors could have an impact on the feasibility of remote pharmaceutical assessments, e.g., the nature of the audit, the characteristics of the audited facility, the quality of the in-country Internet connection, etc.

Therefore, this operational research was conducted to evaluate the feasibility of remote GSDP technical visits of pharmaceutical distributors located in an LMIC hard-to reach during the COVID-19 pandemic. Secondary objectives included describing the challenges, benefits and intrinsic limitations of the remote GSDP technical visits, adapting the QUAMED "on-site" GSDP procedure to the "remote" modality, and formulating recommendations on eligibility criteria, procedures, and tools for remote GSDP technical visits.

\section{Methods}

\section{Study design, procedures and setting}

We conducted four pilot remote technical visits of pharmaceutical distributors, and assessed their feasibility. An overview of the study methodology is provided in Fig. 1.

\section{Development or adaptation of standard operating procedures (SOP)}

We developed the SOP for the remote GSDP assessment. It is based on a previous SOP for on-site GSDP visit, which uses a standardized questionnaire, and a rating system that individually assesses twelve quality assurance activities [2]. The new SOP covers the respective roles and responsibilities of the remote expert and of the incountry assessment facilitator (ICAF), including confidentiality and conflict of interest, the practical setup for the remote technical assessment, including what should happen before it (e.g., sending upfront selected documents, including licenses, organigrams, list of supplied products, etc.), during it (e.g., interviews with key staff, visit of the premises, essential functions to be filmed or photographed etc.), and after it (i.e., the reporting aspects). Importantly, the SOP includes aspects specific to the remote methodology, such as the selection, minimum competencies and training of the in-country assessment facilitator (ICAF). The ICAF, who should be free from any conflict of interest, should be present at the assessed distributor for ensuring the link with the remote expert throughout the remote assessment, e.g., by positioning the video camera as required by the expert, doing translations, double checking on site documents etc.. We also adapted the SOP to address the use of digital technology (video and audio recording, pictures of premises, documents etc.) and the management of information obtained remotely, in compliance with the European Union's General Data Protection Regulation 2016.

\section{Selection of country and distributors}

The essential requirements for selecting the study country were the possibility to use IT communication, and the availability of support from QUAMED member organization(s) (from now onward called "QUAMED member") working in-country, e.g., for selecting and contacting the distributors, appointing an ICAF etc. Countries where English or French are usually accepted working languages were preferred. Furthermore, incountry local distributors were eligible if indicated by a QUAMED member in the country as potential suppliers of medicines and health products.

A call for interest was sent to all QUAMED members in July 2020. The process resulted in the selection of an LMIC hard to reach during the pandemic, where two QUAMED members interested in this work were present, and of four pharmaceutical distributors within it. The four distributors accepted the QUAMED remote assessments. To keep the promise of confidentiality made to them, neither the country nor the distributors are made identifiable in the study report and in this manuscript.

\section{Selection of ICAFs}

To be eligible as ICAF, local staff of QUAMED members operating in the study country needed to be fluent in English or French (depending on the country), to understand local languages (as applicable), and not to have any professional or other links to the assessed distributors. Importantly, they needed to have proven experience in medical supply chain management. Candidates with formal training in pharmacy or logistics, and/or previous knowledge of WHO GSDP were preferred. Potential ICAFs were identified by the QUAMED member(s) operating in the study country, and applications were assessed by the expert. Two experienced ICAFs were selected, a pharmacist, and a logistics manager trained in business administration, and remotely briefed by the expert. Each ICAF supported two out of four remote audits.

The remote assessment matrix is presented in Table 1. 


\section{SUMMARY OF METHODOLOGY}
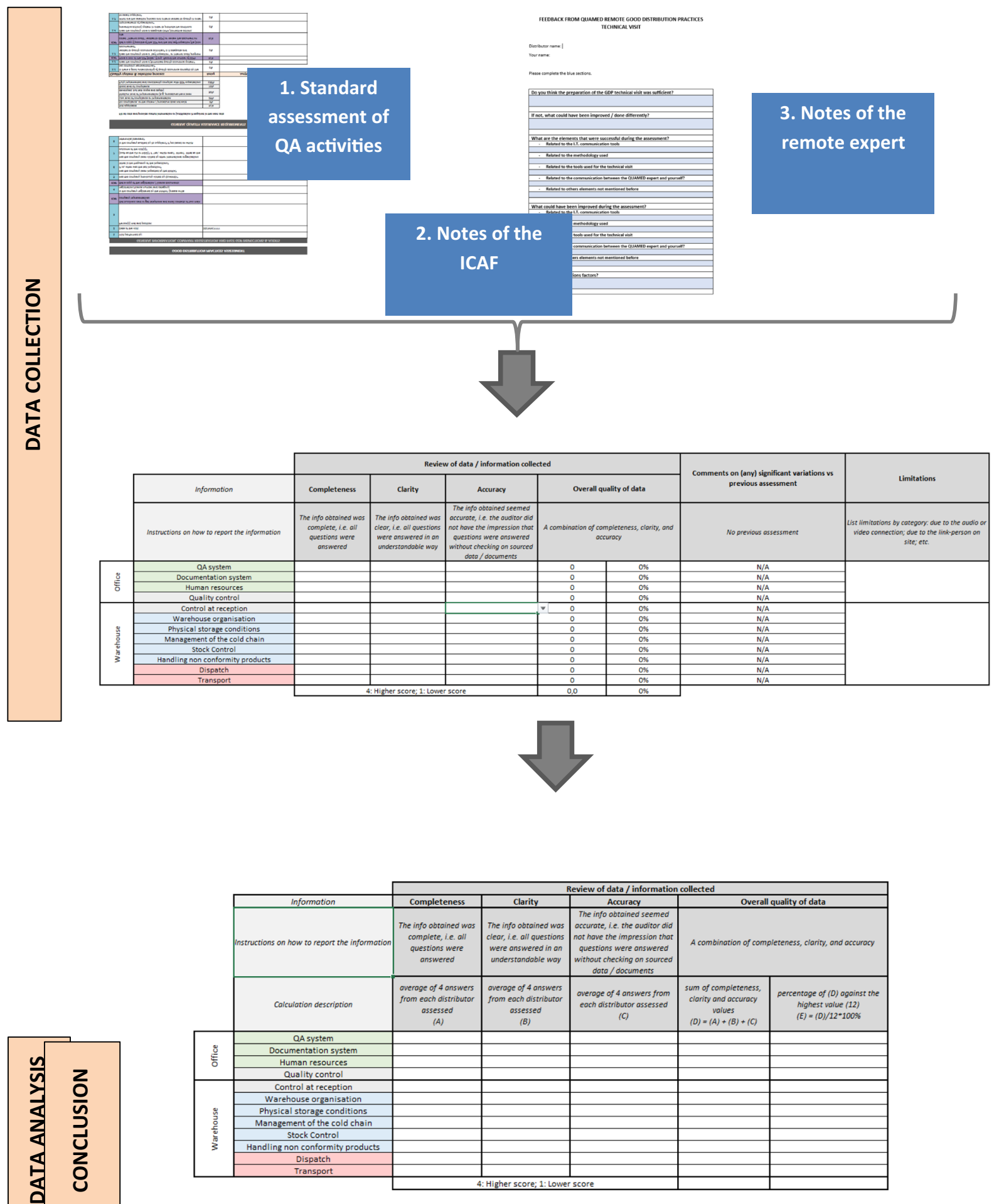

Fig. 1 Summary of methodology 
Table 1 Remote assessment matrix

\begin{tabular}{lllll}
\hline Country & Distributor & Linkage & Expert (remote) & ICAF (onsite) \\
\hline CountryX & Distributor 1 & QUAMED member A & Expert 1 & ICAF alpha \\
Country X & Distributor 2 & QUAMED member B & Expert 1 & ICAF beta \\
Country X & Distributor 3 & QUAMED member A & Expert 1 & ICAF alpha \\
Country X & Distributor 4 & QUAMED member B & Expert 1 & ICAF beta \\
\hline
\end{tabular}

\section{Data collection and analysis}

The remote GSDP technical visits were conducted by the expert between 2nd and 23rd November 2020, with the expert remotely connected and one ICAF physically present onsite. Twelve standard QA activities were assessed by the expert, according to the usual procedure applicable for on-site visits, while the ICAF facilitated the expert's visual access to the relevant spaces, processes and functions. Four out of 12 QA activities (i.e., assessment of the QA system, documentation system, human resources, and quality control) are mainly based on a desk assessment of the documentation provided by the distributor (i.e., office work), while the eight remaining can only be assessed by visiting (seeing) the premises (i.e., remote warehouse assessment). The expert rated each activity for GSDP-compliance. The product sourcing assessment, i.e., an evaluation by the expert of whether the products supplied by the distributors come from reliable manufacturers (based on GMP certification), was conducted by the expert according to the standard procedure as office work. The rating of the QA activities in terms of compliance with the WHO GSDP is separately reported in the QUAMED access-controlled database. The study-specific data were collected by the expert in a "data collection and analysis tool", where the completeness, clarity and accuracy of data collected remotely for each of the 12 QA activities are rated. The information obtained for each activity was rated by the expert in terms of completeness, clarity and accuracy on a $0-4$ scale, where a value above
3.5/4 corresponds to 'excellent', a value between 2.5$3.4 / 4$, to 'good', and a value below $2.5 / 4$, to 'unsatisfactory'. The overall quality per activity, on a $0-12$ scale resulting from the sum of completeness, clarity and accuracy, was assessed as 'excellent' if the overall value was above 10.5 out of 12, as 'good' if between 7.8 and 10.4/12, and 'unsatisfactory' if below 7.7/12. Data were further aggregated for the four distributors. Qualitative notes that helped the expert to formulate the ratings were collected in a "feedback forms" by the ICAFs, and in the notes taken by the expert during the assessment. The data collection and analysis tool is shown in Table 2.

\section{Ethics}

The protocol was approved by the Institutional Review Board of the Institute of Tropical Medicine, Antwerp, Belgium (ref. 1422/20). Distributors freely accepted to be assessed, provided that their confidentiality was protected by not making them, nor the country, identifiable.

\section{Results}

Table 3 presents in aggregated form the results of the expert's assessment of the completeness, clarity and accuracy of QA information obtained remotely for the four distributors. The completeness, i.e., in terms of questions that were answered in the standard QA questionnaire, was excellent ( 3.9 on a $0-4.0$ scale), while the clarity, i.e., the understandability of the information, and

Table 2 Data collection and analysis tool by distributor

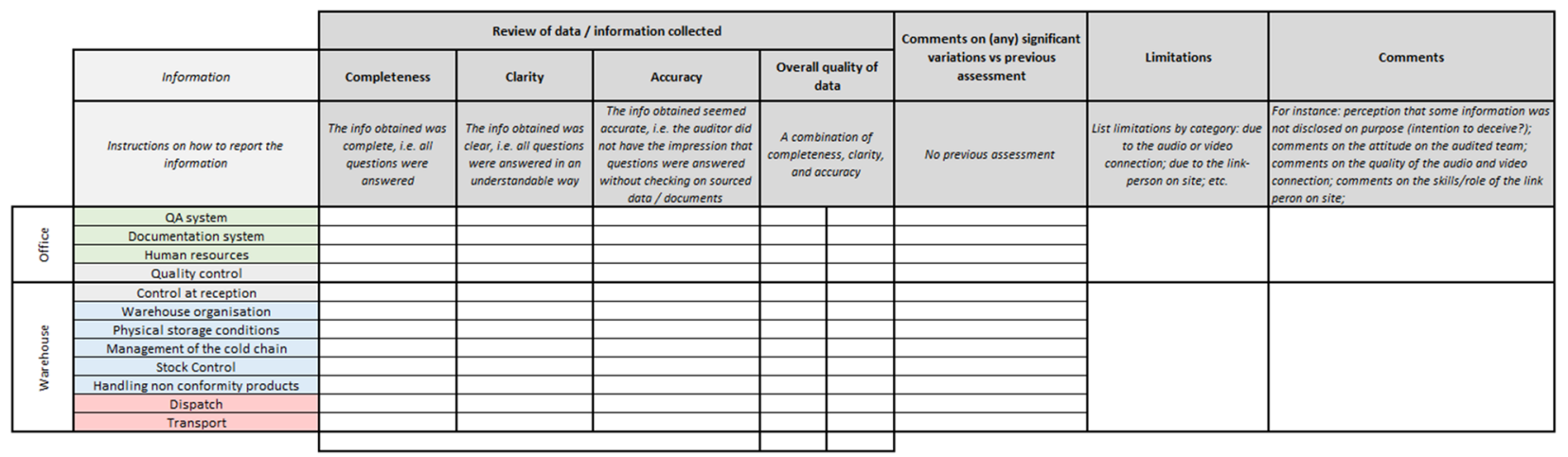


Table 3 Completeness, clarity and accuracy of QA information obtained for GSDP remote assessments across the four distributors

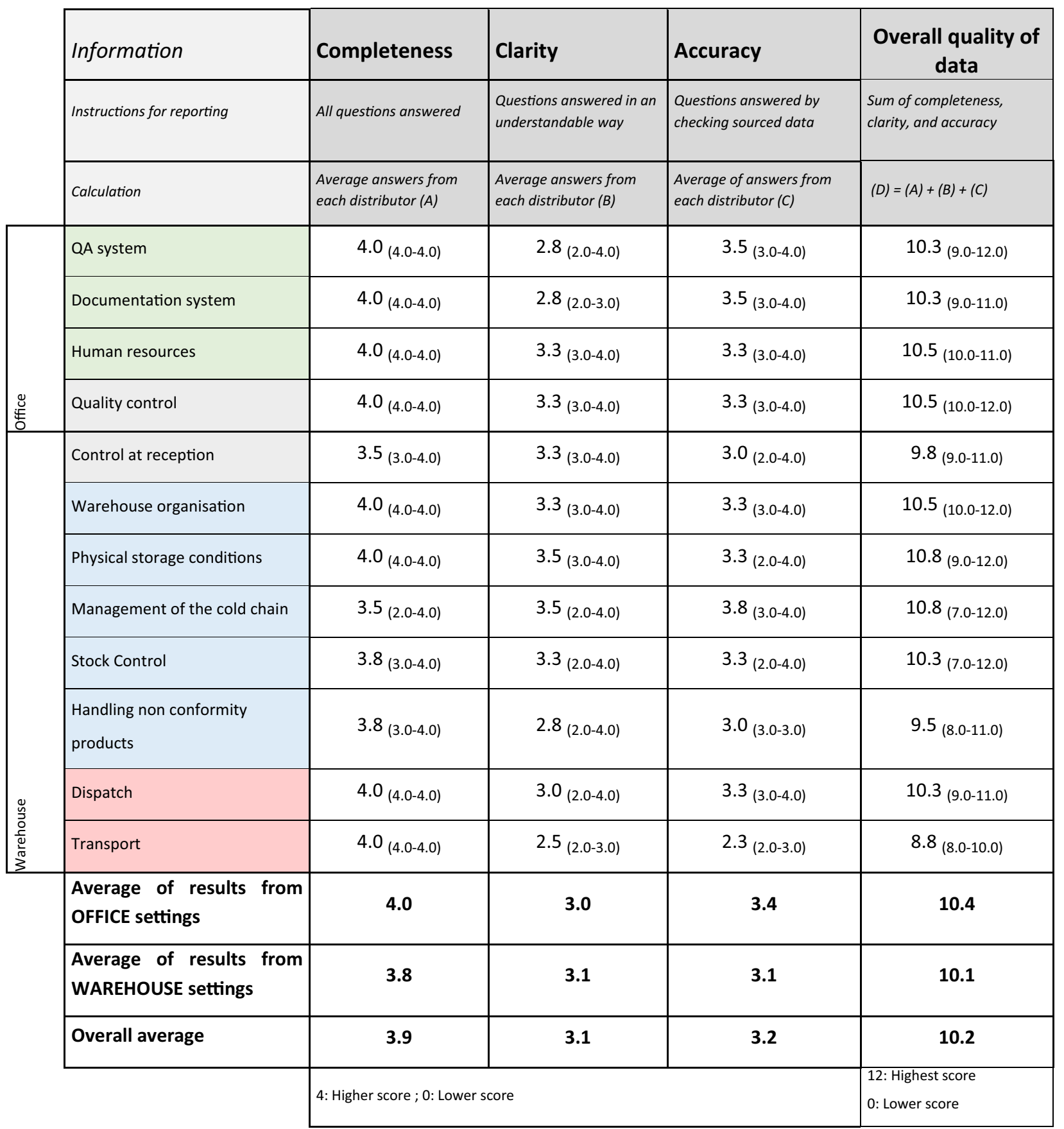

the accuracy, i.e., the perceived reliability of the information, were good (3.1 and 3.2 on a $0-4.0$ scale, respectively). The overall quality of data, calculated by summing completeness, clarity and accuracy, was good (10.2 on a $0-12.0$ scale). As shown in Table 3, there was little variability across distributors.

The difference between the quality of the information prevalently assessed as office work (i.e., desk assessment of documentation on the QA system, documentation system, human resources, and quality control) and the quality of the information assessed during the remote warehouse assessment (i.e., visit of the premises) was small (10.4 versus 10.1$)$. When it comes to the quality of data collected for the 12 specific quality assurance activities, presented in Fig. 2, the lowest values (i.e., less than 10.0) are "handling non-conformity products", "control at 


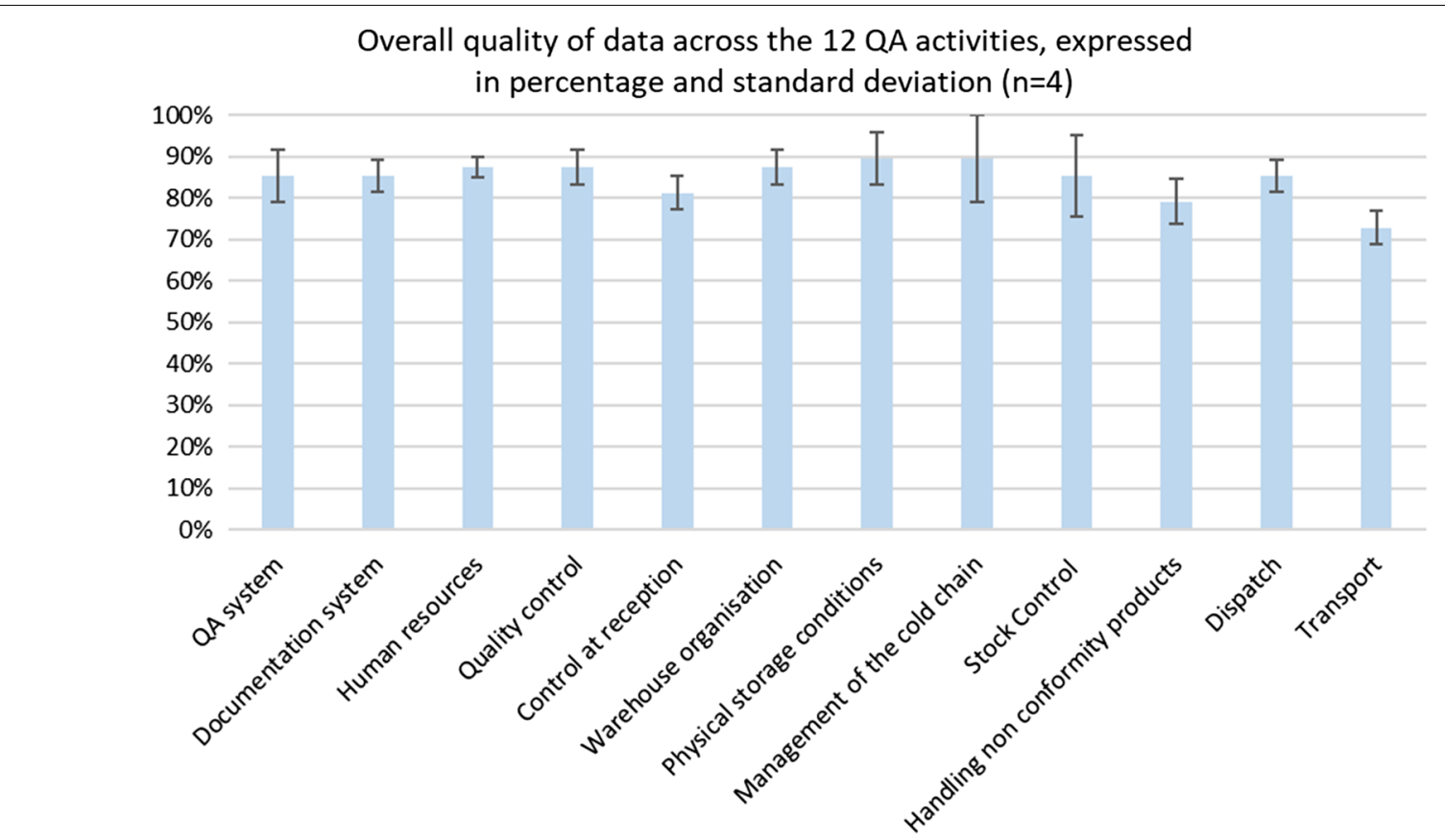

Fig. 2 Overall quality (completeness, clarity, accuracy) of data obtained for the 12 QA activities

reception" and "transport". When it comes to the product sourcing assessment, data provided by the distributors were complete, clear and accurate. This is not surprising, as the product lists provided by email are subject to a desk-assessment only, as it would happen for an on-site visit.

The observed contextual limitations include poor internet connection, particularly in warehouses, requiring frequent repetitions after audio or videoconferencing breaks, some language barriers, and some lack of familiarity in the study country with QA concepts and terminology. Noteworthy, both ICAFs expressed a strong interest to learn more on QA and to stay connected to the study group, after the study.

\section{Discussion}

Some deviations were observed during the field work versus the planned procedures. In particular, we could not compare the findings of this remote audit to the findings of previous onsite audits (available for two out of four distributor), since different data collection tools had been used; the ICAF selection was not done by the QUAMED technical coordinator, but by the expert who would have worked with them, and the study-specific data on clarify, accuracy and completeness were not double checked by a second expert, because this would have lacked essential first-hand experience of the elements observed during the technical visits. Importantly, our findings are exploratory and cannot be extrapolated to other contexts, nor to other types of (more complex) audits (GMP, MQAS etc.). They should be confirmed in different contexts, with different levels of regulatory oversight, to check their replicability. During the next research phases, more tools, such as smart glasses or screen sharing features, could be tested. Furthermore, future research could use more sophisticated designs, for instance, by getting distributors assessed by two different experts in a cross-over sequence, by comparing results obtained at a same distributor with the support of different ICAFs, by adopting a mixed method design to triangulate data from different sources, and-importantly-by validating the methodology by means of comparison of findings from remote and on-site visits conducted at a same distributor.

Despite limitations, data remotely obtained for assessing the GSDP-compliance of 12 essential QA activities in our sample of four distributors was complete, clear and accurate. Variations in overall quality of data were small across distributors (range: 9.9-10.6), across activities (range: 8.8-10.8), and between data obtained for QA activities that require a desk assessment of documentation versus those purely based on the visit of the premises (10.1 versus 10.4). This pilot experience suggests that the intrinsic limitations of remote assessments, e.g., unstable internet connection (particularly in warehouses), languages barrier and impossibility for experts to personally verify premises and documents, can be mitigated by adopting adequate good practices. These include adequate audit 
preparation (e.g., list of documents to be assessed sent to the distributor at least 5 working days upfront, print-outs as well as adequate tools for videoconferencing should be prepared and tested upfront, etc.), ensuring that the "expert and ICAF" pair has language skills adequate to the distributors' context, and planning slightly more time than for an equivalent on-site visit. Furthermore, the role of ICAFs seems crucial for the performance of the remote assessment, as his/ her capacity to guide and orient the remote expert will directly impact the assessment results. Therefore, other key components of the good practices are represented by the individualized training of ICAF, including the essential of GSDP, planning sufficient time and tools to collect the ICAF feedback on the audit, and ICAFs' ability to act as translators as needed. It could also be helpful to have (smartphone) video filmed by a third person, which would allow the expert to see both the ICAF and the distributor representative, during the visit of the warehouse. A careful implementation of these good practices can ensure the feasibility and reliability of remote assessments, to be adopted when an onsite visit is not possible, either because of the pandemic, or of (low-intensity) conflicts, or other reasons.

Furthermore, the interest expressed by ICAFs to keep on working at QA, suggests that remote assessments can create the opportunity to raise awareness among (international and local) staff of organizations that procure medical products about the need to strengthen quality assurance systems, and they can be a starting point to build local capacities. Another potential advantage of the remote assessment is that it can allow to interlink the routine operational activity (assessments) with research activity, given that data collection and analysis can be better tracked and followed. Furthermore, if remote assessments were in the future validated as equivalent to the current gold standard, i.e., the on-site assessments, they would also have a positive environmental impact (we estimate that for this pilot study, there was an approximate savings of 1.2 tons of $\mathrm{CO}_{2}$ emission), they would allow financial savings from spared costs of accommodation, visa fees, per diem, and transport, and they would allow a rapid responsiveness to urgent needs, which can be relatively frequent particularly in humanitarian emergency settings.

For the time being, in addition to being temporarily used instead of on-site assessments when these are unfeasible, remote assessments could also be used as routine tools to complement and strengthen the onsite assessments. For instance, a remote assessment could be set up to pre-screen the distributors proposed for on-site assessment, and avoid unnecessary visits at suppliers which are of very poor quality, with additional savings. Furthermore, the remote methodology could be used for improving the auditor's follow-up of the corrective action plan from onsite visits.

\section{Conclusion}

Our exploratory findings suggest that in case of exceptional circumstances such as the COVID-19 pandemic, a remote assessment of pharmaceutical distributors conducted according to the identified good practices and with the support of a trained ICAF, can provide data of acceptable quality; allow to adequately assess GSDP compliance; and allow to make temporary decisions about licensing (regulators) or purchasing (international organizations, NGOs).

At the moment, on-site assessments remain the gold standards, so the use of remote assessments outside exceptional circumstances and for temporary decisions, would be conditional upon formal validation of this methodology. Meanwhile, the use of remote assessments could be separately considered as part of routine assessments, both for pre-screening candidates for onsite assessments, and for targeted follow-up of on-site assessment.

\section{Abbreviations \\ DMA: Device marketing authorization; EC: European Commission; EEA: Euro- pean economic area; EMA: European Medicines Agency; EU: European Union; FDA: Food and Drug Administration; GMP: Good manufacturing practices; GSDP: Good storage and distribution practices; ICAF: In-country assessment facilitator; IT: Information technology; ITM: Institute of Tropical Medicines, Antwerp; LMIC: Low- and middle-income country; MDCG: Medical Device Coordination Group; MDSAP: Medical Device and Single Audit Program; MQAS: Model of Quality Assurance System for procurement agencies; NRA: National Regulatory Authorities; QA: Quality assurance; QC: Quality control; QCP: Quality Certification Program; SOP: Standard operating procedure; UHC: Universal Health Coverage; USFDA: United States Food and Drug Administra- tion; WHO: World Health Organisation.}

\section{Supplementary Information}

The online version contains supplementary material available at https://doi. org/10.1186/s40545-021-00323-w.

Additional file 1. Study internal report (that contains authors' identifiers).

\section{Acknowledgements}

We are grateful to Ed Vreeke, Executive Director, QUAMED; Corinne Pouget and the other members of the QUAMED Technical Committee; the in-country assessment facilitators; the colleagues of MEDAIR; the staff and management of the assessed distributors. We are thankful to Ariadna Nebot Giralt and Kerlijn Van Assche, for carefully and critically reading our manuscript and giving relevant inputs to improve it.

\section{Authors' contributions}

$A B, L L$ and RR conceptualized the study and wrote the study protocol; $A B$ carried out the study; LL supervised the conduct of the study; RR wrote the first version of this manuscript; $A B$ and $L L$ provided significant input to it. All authors read and approved the final manuscript. 


\section{Funding}

This study was funded by the Belgian Directorate-General for Development Cooperation (DGD) through the Framework Agreement 4, 2017-2021 with the Institute of Tropical Medicines, Antwerp, Belgium.

\section{Availability of data and materials}

Deidentified source data are available in the Study Report, which is provided as Additional file 1 .

\section{Declarations}

\section{Ethics approval and consent to participate}

The protocol was approved by the Institutional Review Board of the Institute of Tropical Medicine, Antwerp, Belgium (ref. 1422/20). Distributors freely accepted to be assessed, provided that their confidentiality was protected by not making them identifiable.

\section{Consent for publication}

Not applicable.

\section{Competing interests}

$R R$ is the Chairperson of the Institutional Review Board of the Institute of Tropical Medicine Antwerp, Belgium, which reviewed and approved the study protocol. She did not participate in the review of this research protocol, which was coordinated by the vice chair.

\section{Author details}

${ }^{1}$ Paris, France. ${ }^{2}$ QUAMED, Brussels, Belgium. ${ }^{3}$ Department of Public Health, Institute of Tropical Medicine, Nationalestraat 155, 2000 Antwerp, Belgium.

Received: 16 February 2021 Accepted: 5 May 2021

Published online: 10 May 2021

\section{References}

1. Ozawa S, Higgins CR, Yemeke TT, Nwokike Jl, Evans L, Hajjou M, et al. Importance of medicine quality in achieving universal health coverage. PLOS ONE. 2020;15(7):e0232966.

2. Nebot Giralt A, Bourasseau A, White G, Pouget C, Tabernero P, Van Assche K, Ravinetto R. Quality assurance systems of pharmaceutical distributors in low-income and middle-income countries: weaknesses and ways forward. BMJ Glob Health. 2020;5:e003147.

3. Seventieth World Health Assembly. Substandard and falsified medical products, 29 May 2017. https://www.who.int/news/item/29-05-2017seventieth-world-health-assembly-update-29-may-2017. Accessed 14 Jan 2021.

4. WHO Global Benchmarking Tool (GBT) for evaluation of national regulatory systems. http://www.who.int/medicines/regulation/benchmarking_ tool/en/. Accessed 14 Jan 2021.

5. Guzman J, O'Connell E, Kikule K, et al. The WHO Global Benchmarking Tool: a game changer for strengthening national regulatory capacity. BMJ Glob Health. 2020;5:e003181. https://doi.org/10.1136/ bmjgh-2020-003181.

6. Perrin C, Cloez S, Dujardin C, Ravinetto R. Europe should lead in coordinated procurement of quality-assured medicines for programmes in lowincome and middle-income countries. BMJ Glob Health. 2020:5:e003283. https://doi.org/10.1136/bmjgh-2020-003283.

7. Official website of QUAMED: https://www.quamed.org/?lang=en. Accessed 14 Jan 2021.

8. World Health Organization, Annex 2: WHO good manufacturing practices for pharmaceutical products: main principles'WHO Technical Report Series No. 986, 2014.

9. World Health Organization. Model quality assurance system for procurement agencies. In: Annex III of the WHO technical report series 986: WHO Expert Committee on Specifications for Pharmaceutical Preparations, forty-eighth report. World Health Organization, 2014.

10. World Health Organization, Annex 7: WHO 'Good storage and distribution practices for medical products', WHO Technical Report Series, No. 1025, 2020.

11. European Medicines Agency. Guidance related to GMP/GDP and PMF distant assessments. EMA/335293/2020. https://www.ema.europa.eu/en/ documents/scientific-guideline/guidance-related-gmp/gdp-pmf-dista nt-assessments en.pdf. Accessed 15 Dec 2020.

12. European Medicines Agency, Guidance on remote GCP inspections during the COVID19 pandemic, EMA/INS/GCP/162006/202018, May 2020 https://www.ema.europa.eu/en/documents/regulatory-proceduralguideline/guidance-remote-gcp-inspections-during-covid-19-pande mic en.pdf. Accessed 7 Jan 2021.

13. Medical Device Coordination Group Document, MDCG 2020-4-European Commission, MDCG 2020-4 https://ec.europa.eu/health/sites/ health/files/md_sector/docs/md_mdcg_2020_4_nb_audits_covid-19_ en.pdf. Accessed 13 Nov 2020

14. Medical device and single audit program transmittal number: 2020-07. https://www.fda.gov/media/136441/download. Accessed 13 Nov 2020

15. https://www.fda.gov/news-events/press-announcements/coronaviruscovid-19-update-fda-prepares-resumption-domestic-inspections-newrisk-assessment-system

16. Medical device coordination group document remote auditing pilot program. MDSAP AU P0036.001 https://www.fda.gov/media/134229/ download. Accessed 13 Nov 2020

17. Durivage M. Remote auditing best practices for GMP compliance. Pharmaceutical Online. Published 25/03/2020. https://www.pharmaceuticalo nline.com/doc/remote-auditing-best-practices-for-gmp-compliance0001 . Accessed 14 Jan 2021

18. Freyr. On-site physical audit to remote audit-a risk-based approach for transition. https://www.freyrsolutions.com/remote-and-virtual-audit. Accessed 07 Apr 2021.

\section{Publisher's Note}

Springer Nature remains neutral with regard to jurisdictional claims in published maps and institutional affiliations.

Ready to submit your research? Choose BMC and benefit from

- fast, convenient online submission

- thorough peer review by experienced researchers in your field

- rapid publication on acceptance

- support for research data, including large and complex data types

- gold Open Access which fosters wider collaboration and increased citations

- maximum visibility for your research: over 100M website views per year

At BMC, research is always in progress.

Learn more biomedcentral.com/submissions 